\title{
Hippocampal TET1 and TET2 Expression and DNA Hydroxymethylation Are Affected by Physical Exercise in Aged Mice
}

\author{
Peter Jessop and Maria Toledo-Rodriguez* \\ School of Life Sciences, University of Nottingham, Nottingham, United Kingdom
}

The function of 5-hydroxymethylcytosine $(5 \mathrm{hmC})$ is poorly understood. $5 \mathrm{hmC}$ is an epigenetic modification of DNA, resulting from the oxidation of 5-methylcytosine $(5 \mathrm{mC})$ by the $\mathrm{Fe}_{2}{ }^{2}$, and 2-oxoglutarate-dependent, 10-11 translocation methylcytosine dioxygenases (TET1, TET2, and TET3). Recent evidence suggests that, in addition to being an intermediate in active demethylation, $5 \mathrm{hmC}$ may also have an epigenetic role. $5 \mathrm{hmC}$ is enriched in the adult brain, where it has been implicated in regulating neurogenesis. The rate of adult neurogenesis decreases with age, however physical exercise has been shown to counteract this deficit. Here, we investigated the impact

OPEN ACCESS

Edited by:

Reinhard Stöger,

University of Nottingham,

United Kingdom

Reviewed by:

lgor Kovalchuk,

University of Lethbridge, Canada

Tomas J. Ekstrom,

Karolinska Institute (KI), Sweden

${ }^{*}$ Correspondence:

Maria Toledo-Rodriguez

maria.toledo@nottingham.ac.uk

Specialty section:

This article was submitted to

Epigenomics and Epigenetics,

a section of the journal

Frontiers in Cell and Developmental

Biology

Received: 01 December 2017

Accepted: 03 April 2018

Published: 19 April 2018

Citation:

Jessop P and Toledo-Rodriguez $M$ (2018) Hippocampal TET1 and TET2

Expression and DNA

Hydroxymethylation Are Affected by

Physical Exercise in Aged Mice.

Front. Cell Dev. Biol. 6:45

doi: 10.3389/fcell.2018.00045 of voluntary exercise on the age-related changes of TET1, TET2, expression and 5hmC content in the hippocampus and hypothalamus. For this purpose, we used voluntary exercise in young adult ( 3 months) and aged (18 months) mice as a rodent model of healthy brain aging. We measured the levels of hippocampal and hypothalamic TET1, TET2 mRNA, and 5hmC and memory [Object Location (OL) test] in mice that either exercised for 1 month or remained sedentary. While aging was associated with decreased TET1 and TET2 expression, voluntary exercise counteracted the decline in expression. Moreover, aged mice that exercised had higher hippocampal $5 \mathrm{hmC}$ content in the promoter region of miR-137, an miRNA involved in adult neurogenesis. Exercise improved memory in aged mice, and there was a positive correlation between $5 \mathrm{hmC}$ miR-137 levels and performance in the OL test. In the hypothalamus neither exercise nor aging affected TET1 or TET2 expression. These results suggest that exercise partially restores the age-related decrease in hippocampal TET1 and TET2 expression, which may be linked to the improvement in memory. Future studies should further determine the specific genes where changes in $5 \mathrm{hmC}$ levels may mediate the exercise-induced improvements in memory and neurogenesis in aged animals.

Keywords: TET1, TET2, 5hmC, exercise, aging, hippocampus

\section{INTRODUCTION}

$5^{\prime}$-methylcytosine $(5 \mathrm{mC})$ is a widely known and functionally well-understood epigenetic DNA modification. Less well-understood are the epigenetic modifications resulting from the iterative oxidation of $5 \mathrm{mC}$ by the $\mathrm{Fe}^{2+}$ and 2-oxoglutarate-dependent dioxygenases: translocation methylcytosine dioxygenases (TET1, TET2, and TET3) (Kriaucionis and Heintz, 2009; Tahiliani et al., 2009). These oxidation products are, sequentially: 5-hydroxymethylcytosine (5hmC) 
(Tahiliani et al., 2009), 5-formylcytosine (5fC), and 5carboxylcytosine (5caC) (Ito et al., 2011). The TET enzymes are thought to be implicated in the active demethylation of DNA, as the enzyme TDG (Thymine-DNA glycosylase) can remove $5 \mathrm{fC}$ and $5 \mathrm{caC}$ from the genome via base excision ( $\mathrm{He}$ et al., 2011). However, evidence suggests that, in addition to being an intermediate in active demethylation, $5 \mathrm{hmC}$ may also have an epigenetic role (Kriaucionis and Heintz, 2009). Interestingly, the highest levels of $5 \mathrm{hmC}$ within adult vertebrates are found in the brain, specifically in neurons (Globisch et al., 2010; Ruzov et al., 2011). The reason for this enrichment is currently unknown, but it may be a consequence of the neurogenic process (Zhang et al., 2013). When comparing neuronal precursor cells (NPCs) and mature neurons, there is a clear increase to $5 \mathrm{hmC}$ levels during differentiation (Zhang et al., 2013). Indeed, the accumulation of $5 \mathrm{hmC}$ in neuronal genes is positively correlated with increases in gene expression (Lin et al., 2017).

Differences to the genomic locations of $5 \mathrm{hmC}$ enrichment indicate that $5 \mathrm{hmC}$ plays multiple roles, varying by tissue type (Globisch et al., 2010) and developmental stage (Ruzov et al., 2011). This is unlike $5 \mathrm{mC}$, which predominantly acts as a transcriptional repressor while, when located in the gene-body, $5 \mathrm{mC}$ can act as a transcriptional activator (Aran et al., 2011; Jones, 2012). For example, there is a stark difference in the genome-wide pattern of $5 \mathrm{hmC}$ between stem cells and brain cells, which might have a functional impact (Ficz et al., 2011; Pastor et al., 2011; Williams et al., 2011; Xu et al., 2011). In mouse embryonic stem cells, $5 \mathrm{hmC}$ is abundant at enhancers (Pastor et al., 2011; Wu et al., 2011; Yu et al., 2012) and at the transcriptional start sites of genes (TSS) (Ficz et al., 2011; Pastor et al., 2011; Williams et al., 2011; Xu et al., 2011). In neurons and brain tissues, $5 \mathrm{hmC}$ tends to be enriched within gene bodies (Xu et al., 2011). Interestingly a significant fraction of neuronal $5 \mathrm{mC}$ and $5 \mathrm{hmC}$ is located at non-CpG sites (where $5 \mathrm{mC}$ is predominantly deposited), namely at CpA dinucleotides (Jang et al., 2017). Additionally, 5hmC appears to play different roles according to the identity of the nucleotide flanking the modified cytosine (Kinde et al., 2015). This suggests that the potential roles of $5 \mathrm{hmC}$ in neurons may be different to other cell types.

In agreement with the high $5 \mathrm{hmC}$ levels in the brain, TET enzyme expression is also enriched (Hahn et al., 2013) and, together with $5 \mathrm{hmC}$, have been shown to play a key role in neuronal differentiation and function (Santiago et al., 2014). For example, Zhang et al. recently showed that mice deficient in TET1, while still viable and fertile, showed decreased adult neurogenesis and impaired spatial learning and short-term memory (Zhang et al., 2013). Interestingly, while this study showed that TET1 is highly expressed in neuronal progenitor cells, the negative effect of TET1 deficiency on neurogenesis was evident only in adulthood. These findings suggest that TET1 and 5hmC might be implicated in maintaining healthy levels of neurogenesis throughout the lifespan. A portion of the $5 \mathrm{mC}$ oxidized by TET enzymes is located within the genes of micro RNAs crucial for adult neurogenesis, such as RNA miR-137. Amongst others, miR-137 regulates the levels of Ezh2, the histone methylase responsible for tri-methylating lysine 27 in histone 3 (H3K27me3), which defines the "poised state" (H3K27me3, H3K4me3) found in many neurogenic genes during neurogenesis (silent genes poised to be activated upon H2K27me3 demethylation).

The aging process is regulated by genetic and environmental factors (Govindaraju et al., 2015). While the genome determines the average life expectancy of a species, environmental factors, via epigenetic changes, regulate differences in longevity between individual members of a species [which can reach up to a 10-fold difference in honeybees and ants, (Kucharski et al., 2008)]. Aging is characterized by an alteration of epigenetic patterns or "epigenetic drift," particularly affecting 5mC (Sierra et al., 2015). During aging there is a parallel genome-wide hypomethylation and promoter-specific hypermethylation (Jung and Pfeifer, 2015). Similarly, recent reports have indicated that there is a global increase of $5 \mathrm{hmC}$ in aged mice (Chen et al., 2012). However, the causal relationship between changes in epigenetic features in the brain, such as $5 \mathrm{hmC}$, and the processes controlling aging are still not clear.

Physical exercise is recommended by the World Health Organization to improve and maintain health throughout the lifespan (World Health Organization, 2010). In the context of brain aging, it is known that exercise counteracts the decline in cognition and neurogenesis in old age (van Praag et al., 2005). Multiple studies have looked at the molecular mechanisms underlying the effects of exercise on brain health (Elsner et al., 2013; Morse et al., 2015). However, to our knowledge, there is no study focusing on the impact of voluntary exercise during aging on brain TET1, TET2 expression, and 5hmC levels.

Here, we have studied the impact of voluntary exercise on the age-related changes to TET1, TET2 expression, and $5 \mathrm{hmC}$ content in two different brain regions; the hippocampus and the hypothalamus. For this purpose, young adult and aged mice were allowed voluntary exercise for 1 month. Afterwards their memory was tested and their hippocampal and hypothalamic TET1, TET2 mRNA and 5hmC levels measured. We found that voluntary exercise counteracts the age-related decrease in TET1 and TET2 expression, improves memory and increases $m i R-137$ $5 \mathrm{hmC}$ levels in the hippocampus of aged mice.

\section{METHODS}

\section{Animals, Exercise, and Object Location Test}

Twenty-nine, C57BL/J6 male mice (fifteen 3 months old and fourteen 18 months old, purchased from Charles River, UK) were allowed to acclimatize to the animal facility for 1 week after arrival. Animals were then single housed and randomly distributed into two groups: exercise (young $n=8$; aged $n=8$ ) and sedentary (young $n=7$; aged $n=6$ ). Mice were on a $12 \mathrm{~h}$ light-dark cycle with food and water available ad libitum. Mice in the exercise group were housed with a running wheel for the entire duration of the study. The distances and time run by each animal were recorded daily. Mice in the sedentary group were housed with a non-functional wheel to control for environmental enrichment. After 4 weeks aged mice underwent the Object Location (OL) test, recognition of a familiar object 
moved to a novel location. This is a very mild behavioral test, which should not produce any stress or long-term changes of gene expression in the brain. Mice were habituated to a novel rectangular arena for $10 \mathrm{~min}$. Afterwards mice were returned to their home cage for $5 \mathrm{~min}$ while the arena was cleaned and two identical objects were set in opposite corners of the same side of the arena. Then mice were returned to the arena and allowed to explore the objects for 6 min (habituation). After the habituation period, the mice were returned to their home cage for $5 \mathrm{~min}$ while the arena and objects were cleaned. One of the objects was placed in the original location while the other was moved to the across the arena (objects in opposite sides of the arena). Animals were returned to the arena and allowed to freely explore the objects for $6 \mathrm{~min}(\mathrm{OL})$. Object investigation was manually recorded using Clicker software (v1.13). The investigation ratio was calculated as the percentage of the total exploration time spent investigating the object in the novel location vs. time exploring both objects. Five weeks after the start of the experiment, mice were humanely killed by cervical dislocation, their brains extracted and then the hippocampus and hypothalamus were dissected and immediately frozen. This study was carried out in accordance with the license authorities issued under the UK Animals (Scientific procedures) Act 1986. The project was approved by the University of Nottingham Animal Welfare and Ethical Review Body.

\section{Gene Expression Profiling by Q-PCR}

RNA was isolated with the AllPrep DNA/RNA/Protein mini Kit (Qiagen, Germany) following the manufacturer's instructions. 500 ng of RNA were reverse transcribed with Superscript III (Life Technologies, UK) and 15-mer random primers (Sigma, $\mathrm{UK})$. Q-PCR reactions were then performed in triplicates with the SensiMix Plus SYBR Green PCR kit (Bioline, UK) in a RotorGene 6000 cycler (Qiagen, Germany). PCR primers for TET1 [NM_001253857.2] [forward primer 5'-ATC ATT CCA GAC CGC AAG AC-3'] and reverse primer 5'-AAT CCA TGC AAC AGG TGA CA-3'] and TET2 [NM_001346736.1] [forward primer 5'-CCA CAG AGA CCA GCA GAA CA-3' and reverse primer $5^{\prime}$-TCC GCT TTC TTC TTG CAA CT$3^{\prime}$ ] were designed with the Primer3 software (http://primer3.ut. ee/). Primer's efficiency and specificity was verified using melting curve analysis (single melt curve peak). For the hippocampus, gene expression was normalized to the geometric average of the control genes [Pgk1, TATA-box binding protein (TBP) and Hprt1] according to the GeNorm normalization (Vandesompele et al., 2002), for the hypothalamus, gene expression was normalized to the control gene $P g k 1$ (as other housekeeping gene candidates were affected by the treatment). Primers for Pgk1 [NM_008828.3] [forward primer 5'-GAA GGG AAG GGA AAA GAT GC-3' and reverse primer $5^{\prime}$-AAA TCC ACC AGC CTT CTG TG-3'], TBP [NM_013684.3] [forward primer 5'-CAG CCT TCC ACC TTA TGC TC- $3^{\prime}$ and reverse primer $5^{\prime}$-TGC TGC TGT CTT TGT TGC TC-3'], and Hprt1 [NM_013556.2] [forward primer 5'-GCA GTA CAG CCC CAA AAT GG-3' and reverse primer 5'-AAC AAA GTC TGG CCT GTA TCC A-3']. The relative expression levels of each mRNA were calculated using a modified 2 Delta-Delta-Ct algorithm (Pfaffl, 2001).

\section{MeDIP}

Genomic DNA was isolated with the AllPrep DNA/RNA/Protein mini Kit (Qiagen, Germany) following the manufacturer's instructions. Quality and quantity of the gDNA was assessed with nanodrop (ThermoFisher, UK). For $5 \mathrm{hmC}$ analysis, $1 \mathrm{ug}$ per sample was sonicated using a Bioruptor (Diagenode, Belgium). Two hundred nanograms from each sample were then removed to serve as an input control before MeDIP was performed. The remaining sonicated gDNA was precipitated using rabbit-anti5hmC antibody (39769, Active Motif, CA, USA) followed by incubation with anti-rabbit Dynabeads (ThermoFisher Scientific, $\mathrm{UK})$. Afterwards, the samples were digested with proteinase $\mathrm{K}$ (P2308, Sigma, UK) followed by phenol-chloroform extraction and ethanol precipitation. Q-PCR reactions were then performed in triplicates with the SensiMix Plus SYBR Green PCR kit (Bioline, UK) in a RotorGene 6000 cycler (Qiagen, Germany). PCR primers for mature miR-137-3p [NM_008173.3] [forward primer $5^{\prime}$-GAT TTA TGG TCC CGG TCA AG-3' and reverse primer 5'-AAT ACC CGT CAC CGA AGA GA-3'] were designed with the Primer3 software (http://primer3.ut.ee/). The $m i R-137$ sequence was obtained from www.mirbase.org This sequence contains the Neuron-Restrictive Silencer Element NRSE/RE1 response element (Warburton et al., 2015), which has been shown to be associated with inactivation and, recently, activation of nonneuronal gene expression (Zhao et al., 2017) (see Figures 2C,D for site and sequence information, respectively). Primer efficiency and specificity were verified using melting curve analysis (single melt curve peak). Enrichment of methylated DNA was calculated using the method detailed by Taiwo et al. (2012). A parallel MeDiP study, using rabbit-anti-5mC antibody (61255, Active Motif, CA, USA), was performed to investigate the impact of exercise on hippocampal $5 \mathrm{mC}$ content.

\section{5hmC ELISA}

Total 5hmC DNA levels were measured using the Quest $5 \mathrm{hmC}$ DNA ELISA Kit (D5425, Zymo Research, USA) following the manufacturer's instructions. In brief, $50 \mathrm{ng}$ from each sample were tested in duplicates or triplicates using a sandwich design. Anti-5hmC antibodies were bound to the ELISA plate, afterwards genomic DNA was incubated followed by anti-DNA HRP conjugated antibodies and developer. The 405-450 nm absorbance was measured on a TECAN "sunrise" basic ELISA model (TECAN, Austria).

\section{Statistics}

Q-PCR and ELISA data were analyzed using two-way ANOVA (for single comparison between groups we used Student's $t$-test). MeDIP and OL results were analyzed using Student's $t$-test. In all cases, normality of the data distribution was confirmed by d'Agostino test. Pearson's correlation was employed to study the relationship between gene expression, $5 \mathrm{hmC}$ or $5 \mathrm{mC}$ levels and OL results. Analyses were performed with Prism (version 6 GraphPad Software, USA). Statistics reported in the text and figures represent the mean \pm S.E.M. For all tests, null hypotheses were rejected at probability level of $p<0.05$. 


\section{RESULTS}

Using a rodent model of long-term voluntary exercise, we studied the impact of physical exercise on memory, $5 \mathrm{mC}$, $5 \mathrm{hmC}$ levels, and TET1 and TET2 expression in the hippocampus and hypothalamus of young adult and aged mice.

\section{Aging Decreased Hippocampal TET1 and TET2 Expression, Which Was Rescued by Physical Exercise}

Both, young and aged mice spontaneously run after getting access to the wheels, increasing their daily running distance until $\sim 10$ days (when they run similar distances day after day). While young mice reached average running distances of $5-15 \mathrm{~km}$ a day, aged mice run distances of $0.5-4 \mathrm{~km}$ a day (data not shown). Aged mice expressed significantly lower levels of TET1 mRNA in their hippocampi $\left[F_{(1,21)}=17.86 p=0.0004\right]$ (Figure 1A). The decrease in TET1 expression was more pronounced in the sedentary aged mice, while it seems that exercise leads to a recovery of TET1 levels in the aged mice. There was a significant age*exercise interaction in TET2 expression $\left[F_{(1,22)}=5.281\right.$ $p=0.0314]$. Post-hoc comparisons indicated that sedentary aged mice had significantly lower TET2 expression than sedentary young mice $\left[t_{(10)}=2.28 p=0.0458\right]$ (Figure 1B) and exerciseaged mice $\left[t_{(11)}=2.673 p=0.0217\right]$ (Figure 1D).

\section{Neither Aging Nor Exercise Affected TET1 or TET2 Expression Levels in the Hypothalamus}

Unlike in the hippocampus, we did not find any significant effect of age or exercise on TET1 or TET2 expression in the hypothalamus (Figures 1C,D). However, there was a tendency for decreased expression of TET2 in aged mice, which seems to be restored by exercise (Figure 2B).

\section{Global Hippocampal 5hmC Levels Increase With Age and Are Negatively Correlated With TET1 Expression}

An increase in TET1 and TET2 expression might result in increased levels of $5 \mathrm{hmC}$ (if $5 \mathrm{mC}$ is oxidized to $5 \mathrm{hmC}$ ) or a decrease in $5 \mathrm{hmC}$ levels (if TET enzymes further oxidize $5 \mathrm{hmC}$ into $5 \mathrm{fC}$ and/or $5 \mathrm{caC}$ ). Using ELISA we measured the total hippocampal $5 \mathrm{hmC}$ content. Aged mice had significantly higher levels of $5 \mathrm{hmC}$ in their hippocampi $\left[F_{(1,21)}=50 p=0.0001\right]$ (Figure 2A). Interestingly, there was a significant negative correlation between the levels of $5 \mathrm{hmC}$ and TET1 expression in the hippocampus. [Pearson's $r_{(21)}=-0.661, p=0.0010$ ] (Figure 2B).

\section{Physical Exercise Significantly Increased Hippocampal 5hmC Content on miR-137 Internal Promoter}

It is known that the impact of aging on $5 \mathrm{mC}$ levels differs depending on the gene. In order to find out whether this applies to $5 \mathrm{hmC}$ we studied the effect of exercise on $5 \mathrm{mC}$ and $5 \mathrm{hmC}$ levels in the internal promoter region of miR-137 in the hippocampus of aged mice (Figure 2B). This region contains the NRSE/RE1 response element (Figures 2C,D). $m i R$ 137 was chosen for this study due to its crucial role in a feedback loop regulating adult neurogenesis (which also includes MeCP2 and Ezh2). Previous research has shown that adult neurogenesis decreases with aging and voluntary exercise counteracts this effect. While voluntary exercise had no effect on the miR-137 $5 \mathrm{mC}$ levels of aged mice $\left[t_{(10)}=0.08454\right.$ $p=0.9343$ ] (Figure 2E), it resulted in a significant increase in the amount of hippocampal miR-137 $5 \mathrm{hmC}\left[t_{(12)}=5.289\right.$ $p=0.0002]$ (Figure 2F). Additionally, we found significant positive correlation between the levels of $5 \mathrm{hmC}$ in miR-137 and TET1 expression in the hippocampus [Pearson's $r_{(12)}=0.598$, $p=0.0400]$ (Figure 2G). The correlation between the levels of $5 \mathrm{hmC}$ in $m i R-137$ and TET2 expression in the hippocampus was not significant [Pearson's $\left.r_{(13)}=0.5244, p=0.0658\right]$ (Figure 2H).

\section{Physical Exercise Significantly Increased Memory (Object Location) in Aged Mice}

Exercise is known to increase adult neurogenesis in aged animals. Increased adult neurogenesis is related to improved memory. Here, we measured the impact of physical exercise on the memory of aged mice using the OL test. Mice that exercise showed a significant increase in the preference index (exploration) of the novel object $\left[t_{(9)}=2.457 p=0.0363\right]$ (Figure 3A). Interestingly, there was a significant positive correlation between the preference index of the mice and their levels of hippocampal miR-137 5hmC [Pearson's $r_{(12)}=0.6965$, $p=0.0119]$ (Figure 3B).

\section{DISCUSSION}

By 2050, worldwide, 1 in 4 people will be 65 years or older (EBioMedicine, 2015). However, this increase in lifespan is not translating into an increase in healthspan (disease free years) (EBioMedicine, 2015). Indeed, with increasing age, the maintenance of good health becomes progressively more challenging, affecting physical as well as mental health. For example, age is the biggest risk factor for the development of neurodegenerative disorders (Niccoli and Partridge, 2012). Maintaining an active lifestyle with regular exercise is known to be beneficial for mental health and, when combined with cognitive training, helps improve neurocognitive function in older adults (Smith et al., 2010; Law et al., 2014). Multiple studies have looked at the molecular mechanisms underlying the effects of exercise on healthy brain aging (Elsner et al., 2013; Morse et al., 2015). However, to our knowledge, no study has focused on the impact of voluntary exercise on expression levels of TET1, TET2, and $5 \mathrm{hmC}$ in young and aged brains.

Here, we have investigated the effects of physical exercise on hippocampal TET1 and TET2 expression during aging. For this purpose, we used: (a) voluntary exercise in young adult (3 months) and aged (18 months) mice as a rodent model of healthy brain aging and (b) Q-PCR to measure gene expression. We compared the age-related changes in animals that exercised or remained sedentary. We showed that exercise in mice caused a 

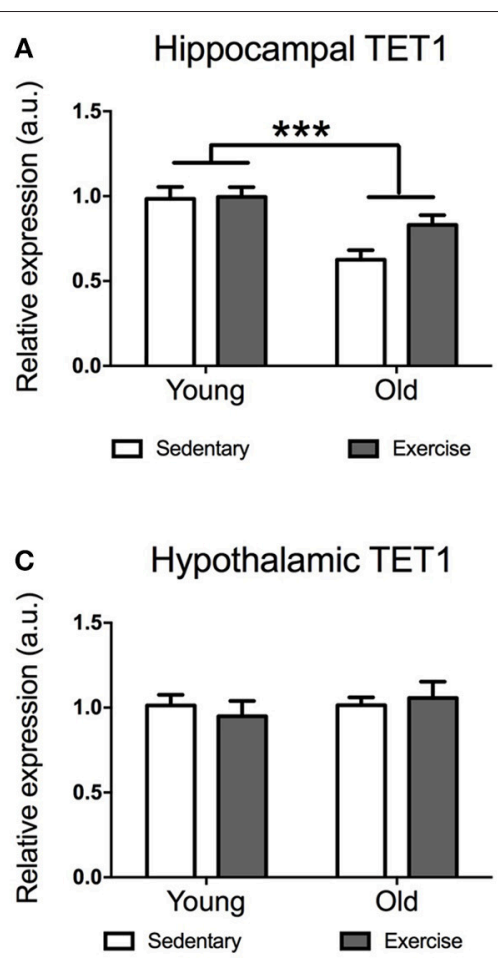
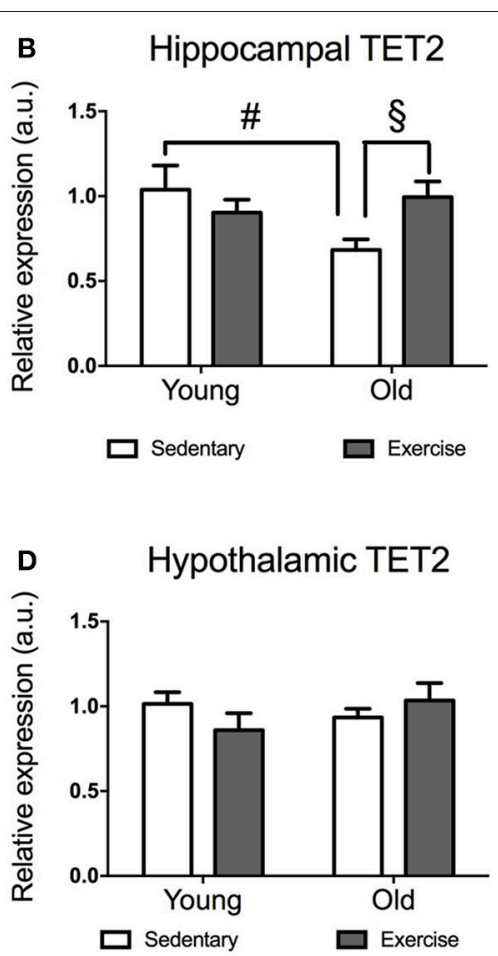

FIGURE 1 | Impact of exercise and aging on TET1, TET2 expression in the hippocampus and hypothalamus. TET1 (A,C) and TET2 (B,D) expression in hippocampus $(\mathbf{A}, \mathbf{B})$ and hypothalamus (C,D) for young and aged mice that exercised or remained sedentary. Young sedentary $n=5-6$, young exercise $n=7-8$, old sedentary $n=$ 5-6, and old exercise $n=7-8 .{ }^{\star \star \star} p<0.001 ;{ }^{\#} p<0.05$ post-hoc comparison between sedentary aged mice and sedentary young mice; ${ }^{\S} p<0.05$ post-hoc comparison between aged mice that exercise and aged mice that remained sedentary.

halt to the age-related decline in TET1 and TET2 expression in the hippocampus, while there were no significant differences in the hypothalamus.

Two previous studies of TET1 and TET2 expression levels in mouse brain tissues suggested a lack of significant changes during postnatal neurodevelopment (Chen et al., 2012; Hadad et al., 2016) however, this data was obtained comparing mice older than our study (24 months old instead of 18 months old). While the age bracket for "old" or "aged" C57BL/6J mice is 18-24 months old, at 24 months there is sharp increase in strain-specific diseases, which can lead to changes in gene expression and thus impact the study outcomes (Flurkey et al., 2007; Moeller et al., 2014). Moreover, both studies use a single housekeeping gene to normalize the Q-PCR results. This can be a confounding factor as, in some, instances the levels of housekeeping gene expression can vary considerably (Suzuki et al., 2000). In our study, we have avoided this by using a combination of three housekeeping genes and GeNorm analysis to compute the normalization factor values, which confirmed that neither age nor exercise affected them (Vandesompele et al., 2002). This strategy enables reliable normalization of Q-PCR data by identifying and employing the most stable control genes to generate a robust normalization factor.

One functional implication of the exercise-induced normalization of hippocampal TET1 and TE2 expression could be changes in adult neurogenesis. Previous studies have shown that TET1 and TET2 are involved in the regulation of neurogenesis (Hahn et al., 2013; Zhang et al., 2013; Santiago et al., 2014) and neuronal activity (Szulwach et al., 2011; Rudenko et al., 2013; Perera et al., 2015). For example, while TET1 knock down does not impact the development or reproduction of mice, it reduces the amount of neuronal progenitor cells in the adult hippocampus and impairs learning and memory (Zhang et al., 2013). Additionally, TET2 knock down results in defective differentiation of newly generated neurons (Hahn et al., 2013). Thus, we could argue the aged-induced downregulation in TET1 and TET2 expression could be implicated in the decrease to neurogenesis in old age. A restoration of TET1 and TET2 expression levels could result in a recovery of adult neurogenesis. This could provide a mechanistic explanation to the exerciseinduced restoration of adult neurogenesis in aged mice (van Praag et al., 2005). Indeed, in this study we showed that aged mice that exercised had improved memory (measured by the OL test) and that the memory (preference index) negatively correlates with the levels of hippocampal $5 \mathrm{hmC}$.

TET1 and TET2 sequentially oxidize $5 \mathrm{mC}$ into $5 \mathrm{hmC}, 5 \mathrm{fC}$, and $5 \mathrm{caC}$ (Tahiliani et al., 2009; Ito et al., 2011). Thus we studied whether the impact of aging and physical exercise on TET1 and TET2 resulted in changes in hippocampal $5 \mathrm{hmC}$ levels. Interestingly we found that aging resulted in a significant increase of hippocampal $5 \mathrm{hmC}$. Indeed, the significant negative correlation between total $5 \mathrm{hmC}$ levels and TET1 expression 
A

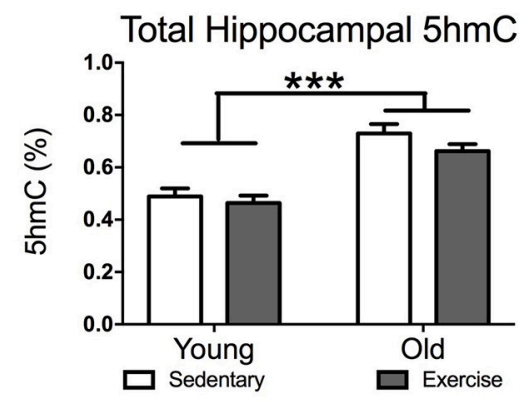

C

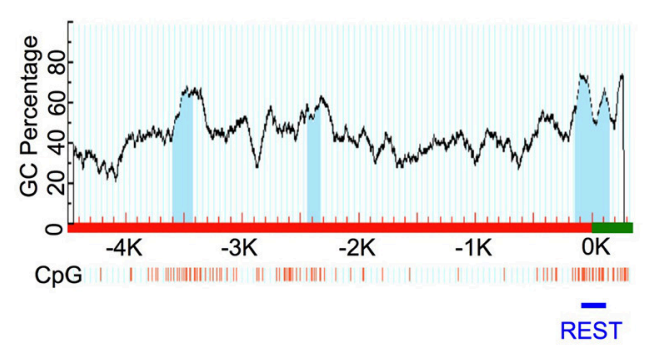

B

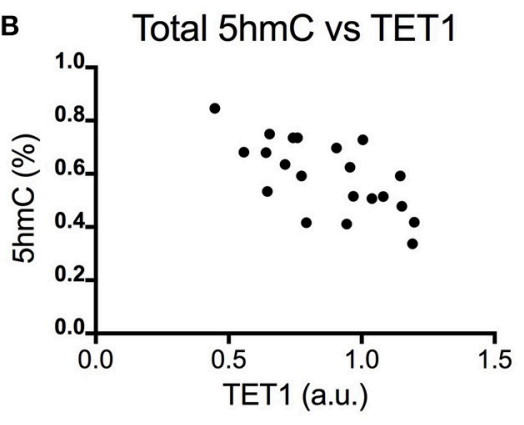

D
E

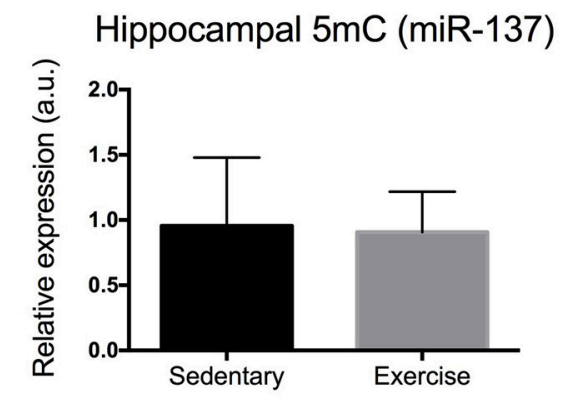

G

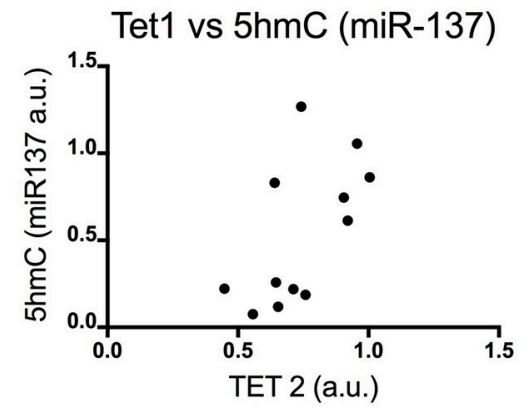

F Hippocampal 5hmC (miR-137)

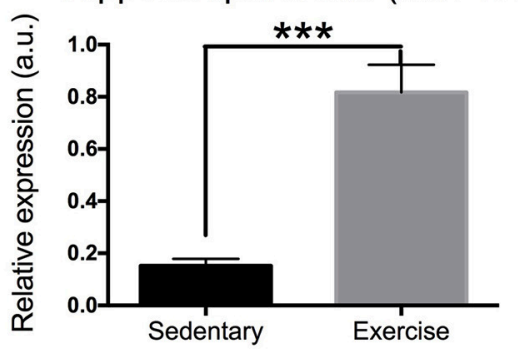

H Tet2 vs $5 \mathrm{hmC}$ (miR-137)

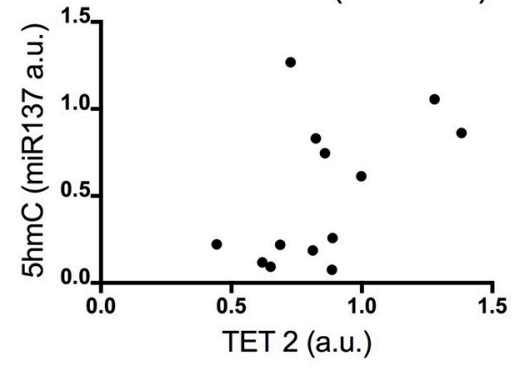

FIGURE 2 | Impact of exercise and aging on total $5 \mathrm{hmC}, 5 \mathrm{mC}(\mathrm{miR}-137)$, and $5 \mathrm{hmC}$ (miR-137) levels in the hippocampus. (A) Total hippocampal 5hmC levels. (B) Correlation between total $5 \mathrm{hmC}$ and TET1 expression in the hippocampus. (C) Scheme of the miR-137 promoter region CpG islands (dark area under the curve) in promoters and $5^{\prime}$-untranslated regions of miR-137 (obtained with http://www.urogene.org/methprimer, individual CpGs are depicted by vertical lines). A horizontal line indicates the region whose DNA methylation was studied by MeDIP. (D) Sequence targeted by PCR primers for the internal miR-137 promoter. (E) $5 \mathrm{mC}$ and (F) $5 \mathrm{hmC}$ enrichment for miR-137 (NRSE/RE1 region) in aged mice that exercised or remained sedentary. Correlation between 5hmC (miR-137) levels and hippocampal expression of (G) TET1 or $\mathbf{( H )}$ TET2 in aged mice. Young sedentary $n=5-6$, young exercise $n=7-8$, old sedentary $n=5-6$, and old exercise $n=7-8$. ${ }^{\star \star \star} p<0.001$.

seems to suggest that the lower levels of TET1 in old animals may result in decreased demethylation. However further studies should investigate whether this is the case. While at first glance, an increase in TET1 expression is expected to result in an increase of $5 \mathrm{hmC}$ levels, that may not be the case if TET1 further oxidizes $5 \mathrm{hmC}$ into $5 \mathrm{fC}$ and $5 \mathrm{caC}$. 


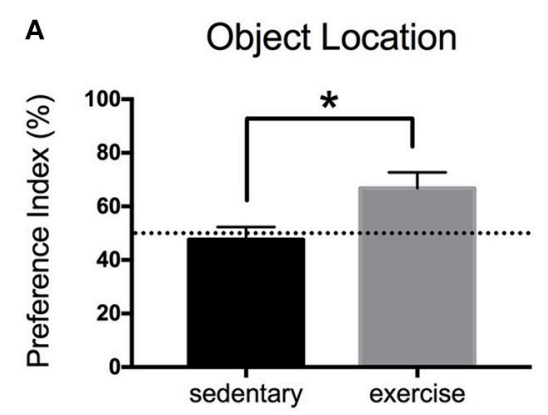

B OL Pref Index vs 5hmC (miR-137)

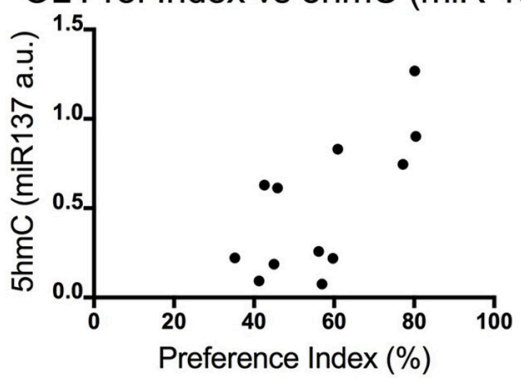

FIGURE 3 | Impact of exercise on memory (OL) in aged mice. (A) Preference index for the OL test for young and aged mice that exercised or remained sedentary. (B) Correlation between hippocampal $5 \mathrm{hmC}\left(\right.$ miR-137) levels and preference index in the OL test. Old sedentary $n=5$ and old exercise $n=6$. ${ }^{*} p<0.05$.

Similar to the impact of aging on $5 \mathrm{mC}$ levels we also found that the impact of aging and exercise on levels of $5 \mathrm{hmC}$ differ depending on the gene studied. The exercise-related changes to TET1 and TET2 expression in aged hippocampi of old mice were accompanied by an approximately 3 -fold increase to the level of $5 \mathrm{hmC}$ at the internal promoter of the neurogenic factor, $m i R-137$. Additionally, the correlation analyses we performed suggest that the degree of hydroxymethylation at the miR-137 promoter region interrogated is higher in hippocampi with higher TET1 and TET2 expressions. Irier et al. recently described that environmental enrichment reduces $5 \mathrm{hmC}$ content in the hippocampus of aged mice (Irier et al., 2014). However, while their environmental enrichment protocol contained a running wheel, the mice only had access to the wheels for $3 \mathrm{~h}$ a day during the light phase of the light/dark cycle. Mice and other nocturnal animals rarely run during the inactive light phase $(0.0-$ $0.1 \mathrm{~km}$ per day vs. $5-15 \mathrm{~km}$ per night for young mice, data not shown) (Goh and Ladiges, 2015). Thus, it is unlikely that the mice in Irier's study did much running (Irier et al., 2014). In our study, mice were housed with the running wheel for a month and thus could run voluntarily during the entire dark phase of the cycle. Moreover, in Irier's study there was only a single running wheel for the 4 mice in the group (Irier et al., 2014). This may have caused fights between the mice and the resulting stress may have confounded the results. In our experiment, each mouse was housed with its own running wheel.

The exercise-induced increase in $5 \mathrm{hmC}$ during aging could mediate, in part, the recovery in adult neurogenesis levels previously reported in aged mice (van Praag et al., 2005). Indeed, in embryonic stem cells, neuronal differentiation is correlated with increase in $5 \mathrm{hmC}$ levels, especially at gene bodies of neuronspecific genes (Hahn et al., 2013). In our study we found that exercise increased $5 \mathrm{hmC}$ content at the internal promoter region of $m i R-137$, a neurogenic miRNA, and this increase is correlated with an improvement in memory. miR-137 is an important regulator of cellular proliferation and differentiation, through regulating translation of the histone methylase, EZH2, thus influencing H3K27 tri-methylation (Mahmoudi and Cairns, 2017). Therefore, change in hydroxymethylation at the miR137 internal promoter could affect neurogenesis levels by modifying miR-137 expression. Additionally, an increase in
miR-137 promoter hydroxymethylation could be implicated in regulating aged-related neuroinflammation. In agreement with this, a recent study has shown that $m i R-137$ overexpression inhibits the inflammatory reaction resulting from spinal cord injury (Gao et al., 2017). The $m i R-137$ region we studied contains the NRSE/RE1 response element (Warburton et al., 2015). The impact of increased $5 \mathrm{hmC}$ levels at the miR-137 NRSE/RE1 region is more difficult to interpret due to the complexity of its ligand's actions (RE-1 Silencing Transcription factor, REST/NRSF). While it is widely known that REST/NRSF regulates the expression of non-neuronal genes through binding to the silencer element NRSE/RE1, recent studies have shown that REST/NRSF is also an activator inducing neuronal differentiation (Zhao et al., 2017). Thus, further studies should explore the functional implications of the increase to hydroxymethylation at the NRSE/RE1 in miR-137.

Understanding whether there is a causative link between the exercise-induced increases to TET1 and TET2 expression, $5 \mathrm{hmC}$ levels and neurogenesis will require further studies. A future experiment could employ the same rodent model of voluntary exercise using homozygous knockout mice for TET1 and/or TET2. Changes in neurogenesis, $m i R-137$ expression and hydroxymethylation patterns at the miR-137 gene could then be examined. As our experiment used the whole hippocampus, future studies in old animals should determine the location and cell type(s) within the hippocampus where TET1, TET2 expression, and $5 \mathrm{hmC}$ level changes take place in response to exercise. Moreover future studies should investigate the differential impact of exercise and aging on $5 \mathrm{hmC}$ in different genes.

\section{AUTHOR CONTRIBUTIONS}

PJ: participated in the design and coordination, carried out the gene expression and MeDIP studies, performed statistical analysis and helped to draft the manuscript; MT-R: conceived the study, participated in the design and coordination, carried out the animal and ELISA studies, performed statistical analysis and drafted the manuscript. All authors read and approved the final manuscript. 


\section{ACKNOWLEDGMENTS}

This work was supported by the Biotechnology and Biological Sciences Research Council [grant number BB/J014508/1] DTP fellowship (PJ); and the University of Nottingham (FRF4294 and

\section{REFERENCES}

Aran, D., Toperoff, G., Rosenberg, M., and Hellman, A. (2011). Replication timingrelated and gene body-specific methylation of active human genes. Hum. Mol. Genet. 20, 670-680. doi: 10.1093/hmg/ddq513

Chen, H., Dzitoyeva, S., and Manev, H. (2012). Effect of aging on 5hydroxymethylcytosine in the mouse hippocampus. Restor. Neurol. Neurosci. 30, 237-245. doi: 10.3233/RNN-2012-110223

EBioMedicine (2015). Increasing healthspan: prosper and live long. EBioMed. 2:1559. doi: 10.1016/j.ebiom.2015.11.015

Elsner, V. R., Lovatel, G. A., Moysés, F., Bertoldi, K., Spindler, C., Cechinel, L. R., et al. (2013). Exercise induces age-dependent changes on epigenetic parameters in rat hippocampus: a preliminary study. Exp. Gerontol. 48, 136-139. doi: 10.1016/j.exger.2012.11.011

Ficz, G., Branco, M. R., Seisenberger, S., Santos, F., Krueger, F., Hore, T. A., et al. (2011). Dynamic regulation of 5-hydroxymethylcytosine in mouse ES cells and during differentiation. Nature 473, 398-402. doi: 10.1038/nature10008

Flurkey, K. M., Currer, J., and Harrison, D. E. (2007). "Chapter 20: Mouse models in aging research," in The-Mouse-in-Biomedical-Research, 2nd Edn., Vol. 3, Normative Biology, Husbandry, and Models, eds M. T. Davisson, F. W. Quimby, S. W. Barthold, C. E. Newcomer, and A. L. B. T. Smith (Burlington, NJ: Academic Press), 637-672.

Gao, L., Dai, C., Feng, Z., Zhang, L., and Zhang, Z. (2017). MiR-137 inhibited inflammatory response and apoptosis after spinal cord injury via targeting of MK2. J. Cell. Biochem. 119, 3280-3292. doi: 10.1002/jcb.26489

Globisch, D., Münzel, M., Müller, M., Michalakis, S., Wagner, M., Koch, S., et al. (2010). Tissue distribution of 5-hydroxymethylcytosine and search for active demethylation intermediates. PLoS ONE 5:e15367. doi: 10.1371/journal.pone.0015367

Goh, J., and Ladiges, W. (2015). "Voluntary wheel running in mice," in Current Protocols in Mouse Biology (Hoboken, NJ: John Wiley \& Sons, Inc.), 283-290.

Govindaraju, D., Atzmon, G., and Barzilai, N. (2015). Genetics, lifestyle and longevity: lessons from centenarians. Appl. Transl. Genomics 4, 23-32. doi: 10.1016/j.atg.2015.01.001

Hadad, N., Masser, D. R., Logan, S., Wronowski, B., Mangold, C. A., Clark, N., et al. (2016). Absence of genomic hypomethylation or regulation of cytosinemodifying enzymes with aging in male and female mice. Epigenetics Chromatin 9:30. doi: 10.1186/s13072-016-0080-6

Hahn, M. A., Qiu, R., Wu, X., Li, A. X., Zhang, H., Wang, J., et al. (2013). Dynamics of 5-Hydroxymethylcytosine and chromatin marks in mammalian neurogenesis. Cell Rep. 3, 291-300. doi: 10.1016/j.celrep.2013.01.011

He, Y.-F., Li, B.-Z., Li, Z., Liu, P., Wang, Y., Tang, Q., et al. (2011). Tet-Mediated formation of 5-carboxylcytosine and its excision by TDG in mammalian DNA. Science 333, 1303-1307. doi: 10.1126/science.1210944

Irier, H., Street, R. C., Dave, R., Lin, L., Cai, C., Davis, T. H., et al. (2014). Environmental enrichment modulates 5-hydroxymethylcytosine dynamics in hippocampus. Genomics 104, 376-382. doi: 10.1016/j.ygeno.2014.08.019

Ito, S., Shen, L., Dai, Q., Wu, S. C., Collins, L. B., Swenberg, J. A., et al. (2011). Tet proteins can convert 5-methylcytosine to 5-formylcytosine and 5-carboxylcytosine. Science 333, 1300-1303. doi: 10.1126/science.1210597

Jang, H. S., Shin, W. J., Lee, J. E., and Do, J. T. (2017). CpG and non-CpG methylation in epigenetic gene regulation and brain function. Genes 8:148. doi: $10.3390 /$ genes 8060148

Jones, P. S. (2012). Functions of DNA methylation: islands, start sites, gene bodies and beyond Nat. Genetics 13, 484-492. doi: 10.1038/nrg3230

Jung, M., and Pfeifer, G. P. (2015). Aging and DNA methylation. BMC Biol. 13:7. doi: 10.1186/s12915-015-0118-4

Kinde, B., Gabel, H. W., Gilbert, C. S., Griffith, E. C., and Greenberg, M. E. (2015). Reading the unique DNA methylation landscape of the brain: non-CpG start-up funds). We would like to thank the Bio Support Unit (University of Nottingham) for their expert and helpful support along the project. We would also like to thank Stephany Villota and Sally Cordon for their assistance with the ELISA experiment and Sam Hurrell for his assistance with the OL analysis.

methylation, hydroxymethylation, and MeCP2. Proc. Natl. Acad. Sci. U.S.A. 112, 6800-6806. doi: 10.1073/pnas.1411269112

Kriaucionis, S., and Heintz, N. (2009). The nuclear DNA base 5hydroxymethylcytosine is present in Purkinje neurons and the brain. Science 324, 929-930. doi: 10.1126/science.1169786

Kucharski, R., Maleszka, J., Foret, S., and Maleszka, R. (2008). Nutritional control of reproductive status in honeybees via DNA methylation. Science 319, 1827-1830. doi: 10.1126/science.1153069

Law, L. L., Barnett, F., Yau, M. K., and Gray, M. A. (2014). Effects of combined cognitive and exercise interventions on cognition in older adults with and without cognitive impairment: a systematic review. Ageing Res. Rev. 15, 61-75. doi: 10.1016/j.arr.2014.02.008

Lin, I. H., Chen, Y. F., and Hsu, M. T. (2017). Correlated 5-hydroxymethylcytosine $(5 \mathrm{hmC})$ and gene expression profiles underpin gene and organ-specific epigenetic regulation in adult mouse brain and liver. PLOS ONE 12:e0170779. doi: 10.1371/journal.pone.0170779

Mahmoudi, E., and Cairns, M. J. (2017). MiR-137: an important player in neural development and neoplastic transformation. Mol. Psychiatry 22, 44-55. doi: $10.1038 / \mathrm{mp} .2016 .150$

Moeller, M., Hirose, M., Mueller, S., Roolf, C., Baltrusch, S., Ibrahim, S., et al. (2014). Inbred mouse strains reveal biomarkers that are pro-longevity, antilongevity or role switching. Aging Cell 13, 729-738. doi: 10.1111/acel.12226

Morse, S. J., Butler, A. A., Davis, R. L., Soller, I. J., and Lubin, F. D. (2015). Environmental enrichment reverses histone methylation changes in the aged hippocampus and restores age-related memory deficits. Biology 4, 298-313. doi: 10.3390/biology4020298

Niccoli, T., and Partridge, L. (2012). Ageing as a risk factor for disease. Curr. Biol. 22, R741-R752. doi: 10.1016/j.cub.2012.07.024

Pastor, W. A., Pape, U. J., Huang, Y., Henderson, H. R., Lister, R., Ko, M., et al. (2011). Genome-wide mapping of 5-hydroxymethylcytosine in embryonic stem cells. Nature 473, 394-397. doi: 10.1038/nature10102

Perera, A., Eisen, D., Wagner, M., Laube, S. K., Künzel, A. F., Koch, S., et al. (2015). TET3 is recruited by REST for context-specific hydroxymethylation and induction of gene expression. Cell Rep. 11, 283-294. doi: 10.1016/j.celrep.2015.03.020

Pfaffl, M. W. (2001). A new mathematical model for relative quantification in real-time RT-PCR. Nucleic Acids Res. 29:e45. doi: 10.1093/nar/29.9.e45

Rudenko, A., Dawlaty, M. M., Seo, J., Cheng, A. W., Meng, J., Le, T., et al. (2013). Tet1 is critical for neuronal activity-regulated gene expression and memory extinction. Neuron 79, 1109-1122. doi: 10.1016/j.neuron.2013.08.003

Ruzov, A., Tsenkina, Y., Serio, A., Dudnakova, T., Fletcher, J., Bai, Y., et al. (2011). Lineage-specific distribution of high levels of genomic 5hydroxymethylcytosine in mammalian development. Cell Res. 21, 1332-1342. doi: 10.1038/cr.2011.113

Santiago, M., Antunes, C., Guedes, M., Sousa, N., and Marques, C. J. (2014). TET enzymes and DNA hydroxymethylation in neural development and function - How critical are they? Genomics 104, 334-340. doi: 10.1016/j.ygeno.2014. 08.018

Sierra, M., Fernández, A., and Fraga, M. F. (2015). Epigenetics of aging. Epigenetics Aging 16, 435-440. doi: 10.2174/1389202916666150817203459

Smith, P. J., Blumenthal, J. A., Hoffman, B. M., Cooper, H., Strauman, T. A., WelshBohmer, K., et al. (2010). Aerobic exercise and neurocognitive performance: a meta-analytic review of randomized controlled trials. Psychosom. Med. 72, 239-252. doi: 10.1097/PSY.0b013e3181d14633

Suzuki, T., Higgins, P. J., and Crawford, D. R. (2000). Control selection for RNA quantitation. Biotechniques 29, 332-337.

Szulwach, K. E., Li, X., Li, Y., Song, C. X., Wu, H., Dai, Q., et al. (2011). 5-hmCmediated epigenetic dynamics during postnatal neurodevelopment and aging. Nat. Neurosci. 14, 1607-1616. doi: 10.1038/nn.2959 
Tahiliani, M., Koh, K. P., Shen, Y., Pastor, W. A., Bandukwala, H., Brudno, Y., et al. (2009). Conversion of 5-methylcytosine to 5-hydroxymethylcytosine in mammalian DNA by MLL partner TET1. Science 324, 930-935. doi: $10.1126 /$ science.1170116

Taiwo, O., Wilson, G. A., Morris, T., Seisenberger, S., Reik, W., Pearce, D., et al. (2012). Methylome analysis using MeDIP-seq with low DNA concentrations. Nat. Protoc. 7, 617-636. doi: 10.1038/nprot.2012.012

Vandesompele, J., De Preter, K., Pattyn, F., Poppe, B., Van Roy, N., De Paepe, A., et al. (2002). Accurate normalization of real-time quantitative RT-PCR data by geometric averaging of multiple internal control genes. Genome Biol. 3, 34-31. doi: 10.1186/gb-2002-3-7-research0034

van Praag, H., Shubert, T., Zhao, C., and Gage, F. H. (2005). Exercise enhances learning and hippocampal neurogenesis in aged mice. J. Neurosci. 25, 8680-8685. doi: 10.1523/JNEUROSCI.1731-05.2005

Warburton, A., Breen, G., Rujescu, D., Bubb, V. J., and Quinn, J. P. (2015). Characterization of a REST-regulated internal promoter in the schizophrenia genome-wide associated gene MIR137. Schizophr. Bull. 41, 698-707. doi: 10.1093/schbul/sbu117

Williams, K., Christensen, J., Pedersen, M. T., Johansen, J. V., Cloos, P. A., Rappsilber, J., et al. (2011). TET1 and hydroxymethylcytosine in transcription and DNA methylation fidelity. Nature 473, 343-348. doi: 10.1038/nature10066

World Health Organization (2010). Global Recommendations on Physical Activity for Health.

Wu, H., D’Alessio, A. C., Ito, S., Wang, Z., Cui, K., and Zhao, K. (2011). Genomewide analysis of 5-hydroxymethylcytosine distribution reveals its dual function in transcriptional regulation in mouse embryonic stem cells. Genes Dev. 25, 679-684. doi: 10.1101/gad.2036011
Xu, Y., Wu, F., Tan, L., Kong, L., Xiong, L., Deng, J., et al. (2011). Genome-wide regulation of $5 \mathrm{hmC}, 5 \mathrm{mC}$, and gene expression by Tet1 hydroxylase in mouse embryonic stem cells. Mol. Cell 42, 451-464. doi: 10.1016/j.molcel.2011.04.005

Yu, M., Hon, G. C., Szulwach, K. E., Song, C. X., Zhang, L., Kim, A., et al. (2012). Base-resolution analysis of 5-hydroxymethylcytosine in the mammalian genome. Cell 149, 1368-1380. doi: 10.1016/j.cell.2012.04.027

Zhang, R. R., Cui, Q. Y., Murai, K., Lim, Y. C., Smith, Z. D., Jin, S., et al. (2013). Tet1 regulates adult hippocampal neurogenesis and cognition. Cell Stem Cell 13, 237-245. doi: 10.1016/j.stem.2013.05.006

Zhao, Y., Zhu, M., Yu, Y., Qiu, L., Zhang, Y., He, L., et al. (2017). Brain REST/NRSF is not only a silent repressor but also an active protector. Mol. Neurobiol. 54, 541-550. doi: 10.1007/s12035-015-9658-4

Conflict of Interest Statement: The authors declare that the research was conducted in the absence of any commercial or financial relationships that could be construed as a potential conflict of interest.

The handling Editor declared a shared affiliation, though no other collaboration, with the authors PJ, MT-R.

Copyright (c) 2018 Jessop and Toledo-Rodriguez. This is an open-access article distributed under the terms of the Creative Commons Attribution License (CC $B Y)$. The use, distribution or reproduction in other forums is permitted, provided the original author(s) and the copyright owner are credited and that the original publication in this journal is cited, in accordance with accepted academic practice. No use, distribution or reproduction is permitted which does not comply with these terms. 